\title{
Gender and international clinician educators
}

\author{
Dora J Stadler, ${ }^{1}$ Sophia Archuleta, ${ }^{2,3}$ Halah Ibrahim, ${ }^{4}$ Nina G Shah, ${ }^{5}$ \\ Ahmed Ali Al-Mohammed, ${ }^{6,7}$ Joseph Cofrancesco $\mathrm{Jr}^{8}$
}

'Continuing Professional Development, Weill Cornell Medicine-Qatar, Doha, Qatar 'Division of Infectious Diseases, National University Hospital, National University Health System, Singapore, Singapore ${ }^{3}$ Yong Loo Lin School of Medicine, National University of Singapore, Singapore, Singapore ${ }^{4}$ Masters of Education for Health Professionals, Johns Hopkins School of Education, Baltimore, USA

${ }^{5}$ Division of Unintentional Injury Prevention, National Center for Injury Prevention and Control, Centers for Disease Control and Prevention, Atlanta, USA

${ }^{6}$ Internal Medicine Residency Program, Hamad Medical Corporation, Doha, Qatar 7 Medical Education, Weill Cornell Medicine-Qatar, Doha, Oatar

${ }^{8}$ Department of Medicine and Institute for Excellence in Education, Johns Hopkins University School of Medicine, Baltimore, Maryland, USA

Correspondence to Dr Joseph Cofrancesco Jr, Department of Medicine, Johns Hopkins University School of Medicine, Armstrong Medical Education, Building 1600, McElderry Street, Suite 230, Baltimore, MD 21205, USA; joeco@jhmi.edu

$\mathrm{SA}$ and $\mathrm{HI}$ contributed equally.

Received 20 October 2016 Revised 16 February 2017 Accepted 5 March 2017 Published Online First 31 March 2017

\section{Linked}

- http://dx.doi.org/10.1136/ postgradmedj-2017-134931

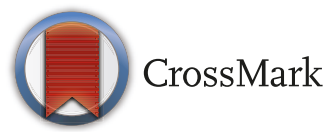

To cite: Stadler DJ

Archuleta S, Ibrahim $\mathrm{H}$, et al. Postgrad Med J

2017:93:719-724.

\section{ABSTRACT}

Objectives To describe gender differences of international clinician educators (CEs) and leaders, and CEs' perceptions by gender of preparation, roles, rewards and factors affecting job satisfaction and retention in emerging international competency-based residency programmes. Methods Cross-sectional surveys of CEs and leadership were conductedJune 2013-June 2014 at institutions that had adopted competency-based graduate medical education and were accredited by the Accreditation Council for Graduate Medical Education-International. Results $274(76.3 \%)$ of 359 eligible participants responded; 69 (25.2\%) were female. Two (18\%) of 11 chief executive officers and $1(9 \%)$ of 11 chief medical officers were women. Female CEs were younger, more likely to be single and childless. They were less likely to hold academic appointments, despite no gender differences in length of time at current institution or in current position. A greater proportion of female CEs felt they were 'never' rewarded by academic promotion. Satisfaction rates were similar between the genders. Single female CEs were five times as likely to report being 'extremely likely' to stay in the country. Female CEs with children $<21$ were less likely to report high likelihood of staying in academia. Marital status and children were not associated with outcomes for male CEs.

Conclusions In the international academic medicine programmes studied, there were fewer female CEs in the pipeline and they perceived a gender gap in appointment and advancement. Stakeholders at international programmes need to develop contextualised strategies to expand entry and decrease attrition of women into CE tracks, and promote gender equity.

\section{INTRODUCTION}

Despite increasing numbers of female medical school graduates ${ }^{12}$ and female faculty members, ${ }^{1}$ there is a growing body of literature describing gender inequality in academic medicine. ${ }^{13-7}$ In the USA and Europe, disparities have been noted in academic rank, career advancement, leadership opportunities and compensation. ${ }^{16-11}$ Differences in scholarly productivity in the sciences have also been observed, ${ }^{12}$ with several studies confirming this discrepancy in the medical field. ${ }^{11}$ While separate clinician educator (CE) tracks have recently developed in the USA, with large numbers of female faculty, ${ }^{13}$ the promotion and recognition of faculty on these tracks are still evolving and lag behind more traditional paths. ${ }^{14} 15$ The issue of gender inequality in academic medicine has been a subject of study for over three decades, with most studies focusing on the recruitment and retention of women in academia. ${ }^{16} 17$ Authors have attempted to identify barriers to success, ${ }^{18}$ as well as gender differences in professional experiences, and perceptions of the culture of academic medicine. ${ }^{19} 20$ Equal access, work-life balance, mentorship opportunities, supportive institutional leadership and a conducive institutional culture ${ }^{2122}$ have emerged as key contributors to the success and career satisfaction of women in academic medicine. International studies from non-Western regions, though far fewer in number, seem to confirm the existence of a gender gap in academic medicine in terms of faculty numbers, promotion rates, research productivity and women's reported perceptions of bias. ${ }^{16} 23-25$ Existing research, however, has not focused on issues relevant to international CEs or graduate medical education (GME).

We previously published results from our multinational Study of Perspectives of International Clinician Educators (SPICE) describing CEs in countries that have recently undergone GME reform to competency-based (CB) education as evidenced by Accreditation Council for Graduate Medical Education International (ACGME-I) certification. ${ }^{26}$ At the time of the study, only Singapore, Qatar and the United Arab Emirates (UAE) had received accreditation. As learners and faculty are increasingly travelling outside their home countries to receive GME training and pursue employment opportunities, ${ }^{27}$ and institutions and countries are making significant investments to train and develop academic faculty, it is paramount to understand the impact of gender in international academic medicine. In this study, we describe issues related to gender in these international academic medical centres. We also describe the association of gender on CEs' perceptions of career satisfaction, work-life balance and likelihood of remaining in academic medicine.

\section{METHODS}

SPICE study methods, including instrument development, have been described previously. ${ }^{26}$ Pertinent to this study, we conducted cross-sectional surveys of CEs at institutions accredited by ACGME-I as of 1 June 2013, including academic medical centres and their affiliated teaching hospitals in Qatar, Singapore and the UAE. Eligible CEs, defined as 'physicians whose primary responsibilities are patient care and education, and whose research represents only minor portion of academic contributions $^{14}$ included programme directors (PD), associate programme directors (APD) and core faculty in CB GME programmes with greater than six residents. Institutional leaders were defined as chief executive officers (CEO), chief medical 
officers (CMO), designated or associated designated institutional officers or their equivalents.

\section{Ethical approval}

The study received approval from the institutional review boards of Johns Hopkins University School of Medicine in Baltimore, Hamad Medical Corporation and Weill Cornell Medicine in Qatar, National University of Singapore and the Al Ain Research Ethics Committee of the UAE. We also received approval from the GME office of each participating site.

\section{Data collection}

The data collection method was previously described. ${ }^{26}$ Briefly, we sent email invitations with electronic links to eligible CEs via SurveyMonkey followed by paper-based surveys to non-responders. A study coordinator in the USA randomly assigned a unique identifier to each survey, but this information was not shared with the study team. Leaders' gender information was collected from publicly available institution websites. All data were exported to the team statistician using Microsoft Excel 2010 .

\section{Data analysis}

We used descriptive statistics to summarise CE characteristics overall and stratified by gender. Covariates of interest included demographics, number and age of children, location of medical school, residency and fellowship training (North America, Europe or other country), institutional affiliation with medical school, academic appointment, current position (ie, PD), years working at current institution, percentage of time spent in certain activities (ie, teaching, patient care), self-reported level of satisfaction, and ideal and actual rewards for time and effort spent teaching. We also examined departments as grouped into standard ACGME categories of medical, surgical or other hospital-based specialties. ${ }^{28}$ Categorical covariates were compared withgender using $\chi^{2}$ tests, and means of continuous covariates were compared with gender using t tests. Outcomes of job satisfaction, likelihood of staying at the institution and in academic medicine were modelled using multivariate logistic regression. Covariates with statistical significance at levels of $\alpha \leq 0.10$ in the bivariate models were entered into the multivariate models. The covariate effects were expressed as OR, adjusted ORs (aOR) and 95\% CI. SAS statistical software V.9.3 (SAS Institute) was used for analyses.

\section{RESULTS}

Three academic institutions in Singapore, one in Qatar and five in the UAE received institutional accreditation by the ACGME-I by the close of the data collection on 30 June 2014. From the eight participating institutions (one Singapore institution declined to participate), $274(74.3 \%)$ of the CEs completed surveys, of whom $205(74.8 \%)$ were men and 69 (25.2\%) were women. This included physicians from eight academic medical centres and two affiliated teaching hospitals, representing 47 residency programmes across 17 specialties. Table 1 shows institutional leadership distribution.

\section{Characteristics of CEs by gender}

The gender breakdown and demographic characteristics of the participating CEs are shown in table 2. Women comprised 25.2\% $(n=69)$ of the workforce, with over half $(53.6 \%, n=37)$ aged 40-49. Overall, females CEs were more likely to be younger, single and without children than their male counterparts. There
Table 1 Institutional leadership by gender

\begin{tabular}{lcl}
\hline $\begin{array}{l}\text { Institutional leadership } \\
\text { position, } \mathbf{n = 3 4}\end{array}$ & Males, $\mathbf{n = 2 6}$ & Females, $\mathbf{n = 8}$ \\
\hline & $\mathrm{n}(\%)$ & $\mathrm{n}(\%)$ \\
Chief executive officer & $9(82)$ & $2(18)$ \\
Chief medical officer & $10(91)$ & $1(9)$ \\
DIO/associate DIO & $7(58)$ & $5(42)$ \\
\hline
\end{tabular}

DIO, designated institutional official.

were no significant differences in location of training for residency, but men were more likely to have received fellowship training in the USA or Canada. Women were more likely to be in medical specialties, as compared with surgical or hospital-based specialties. Within surgery, there were significantly more males in surgical specialties outside of obstetrics-gynaecology. There were no significant gender differences in time at current institution or in current position, or in terms of programme leadership (PD or APD). Though the vast majority of both men and

\begin{tabular}{|c|c|c|c|}
\hline \multirow[t]{2}{*}{ Characteristic } & Males, $n=205$ & Females, $n=69$ & $\mathrm{p}$ Value \\
\hline & $\mathrm{n}(\%)$ & $\mathrm{n}(\%)$ & \\
\hline Age, years & & & $0.002^{*}$ \\
\hline $30-39$ & $30(14.8)$ & $23(33.3)$ & $<0.001$ \\
\hline $40-49$ & $120(59.1)$ & $37(53.6)$ & 0.48 \\
\hline $50-59$ & $41(20.2)$ & $5(7.3)$ & 0.014 \\
\hline $60+$ & $12(5.9)$ & $4(5.8)$ & 0.99 \\
\hline Marital status & & & $<0.001^{*}$ \\
\hline Single & $16(7.9)$ & $19(28.0)$ & $<0.001$ \\
\hline Married/partnered & 185 (91.1) & 47 (69.1) & $<0.001$ \\
\hline Divorced/widowed & $2(1.0)$ & $2(2.9)$ & 0.25 \\
\hline Children & & & $<0.001^{*}$ \\
\hline None & $14(8.4)$ & $14(28.0)$ & \\
\hline Less than 21 years & $153(91.6)$ & $36(72.0)$ & \\
\hline $\begin{array}{l}\text { Residency/postgrad } \\
\text { training }\end{array}$ & & & $0.59^{*}$ \\
\hline USA/Canada & $51(24.9)$ & $13(18.8)$ & 0.31 \\
\hline UK/Europe & 35 (17.1) & $13(18.8)$ & 0.74 \\
\hline Other & $119(58.0)$ & $43(62.3)$ & 0.53 \\
\hline Fellowship training & & & $0.046^{*}$ \\
\hline USA/Canada & $63(30.7)$ & $11(15.9)$ & 0.10 \\
\hline UK/Europe & $38(18.5)$ & $13(18.8)$ & 0.96 \\
\hline Other & $104(50.7)$ & $45(65.2)$ & 0.037 \\
\hline \multicolumn{4}{|l|}{ ACGME specialties } \\
\hline Hospital based & 44 (24.6) & $11(18.3)$ & 0.34 \\
\hline Medical & $81(45.2)$ & $38(63.3)$ & 0.013 \\
\hline Surgical & $54(30.2)$ & $11(18.3)$ & 0.08 \\
\hline $\begin{array}{l}\text { Surgical specialty, } \\
\text { excluding OB/GYN }\end{array}$ & $49(90.7)$ & $4(36.4)$ & $<0.001^{*}$ \\
\hline OB/GYN & $5(9.3)$ & $7(63.6)$ & \\
\hline $\begin{array}{l}\text { Country of current } \\
\text { employment }\end{array}$ & & & $0.12^{*}$ \\
\hline Qatar & $59(28.8)$ & $19(27.5)$ & 0.84 \\
\hline Singapore & $94(45.9)$ & $24(34.8)$ & 0.11 \\
\hline $\begin{array}{l}\text { United Arab } \\
\text { Emirates }\end{array}$ & $52(25.4)$ & $26(37.7)$ & 0.05 \\
\hline
\end{tabular}

${ }^{*} \chi^{2} p$ value is for the whole group comparison.

ACGME, Accreditation Council for Graduate Medical Education; GYN, gynecology; $\mathrm{OB}$, obstetrics. 
Table 3 Faculty appointment/position of international clinician educators by gender

\begin{tabular}{|c|c|c|c|}
\hline Appointment/position & Males, $n=205$ & Females, $n=69$ & p Value \\
\hline & $\mathrm{n}(\%)$ & $\mathrm{n}(\%)$ & \\
\hline $\begin{array}{l}\text { School of medicine } \\
\text { appointment }\end{array}$ & & & $<0.001^{*}$ \\
\hline No appointment & $56(27.3)$ & $34(50.8)$ & \\
\hline Appointment & $149(72.7)$ & $33(49.2)$ & \\
\hline Faculty rank & & & $0.35^{*}$ \\
\hline Instructor & $34(16.6)$ & $6(9.0)$ & 0.13 \\
\hline Assistant professor & $75(36.6)$ & $21(31.3)$ & 0.44 \\
\hline Associate professor & $31(15.1)$ & $6(9.0)$ & 0.20 \\
\hline Professor & $9(4.4)$ & 0 & 0.12 \\
\hline Current position & & & $0.53^{*}$ \\
\hline Programme director & $40(19.5)$ & $15(22.1)$ & 0.65 \\
\hline $\begin{array}{l}\text { Associate programme } \\
\text { director }\end{array}$ & $36(17.6)$ & $15(22.1)$ & 0.41 \\
\hline $\begin{array}{l}\text { Teaching faculty, other } \\
\text { position }\end{array}$ & $129(62.9)$ & $38(55.9)$ & 0.30 \\
\hline \multicolumn{4}{|l|}{$\begin{array}{l}\text { Years at current } \\
\text { institution }\end{array}$} \\
\hline Mean (SD) & $9.3(6.5)$ & $9.2(6.0)$ & 0.89 \\
\hline $\begin{array}{l}\text { Median (first, third } \\
\text { IQR) }\end{array}$ & $8(4,13)$ & $8(5,14)$ & 0.95 \\
\hline \multicolumn{4}{|l|}{ Years in current position } \\
\hline Mean (SD) & $4.2(3.3)$ & $3.6(3.2)$ & 0.20 \\
\hline $\begin{array}{l}\text { Median (first, third } \\
\text { IQR) }\end{array}$ & $3(2,5)$ & $3(1.5,5)$ & 0.08 \\
\hline
\end{tabular}

${ }^{*} \chi^{2} p$ value is for the whole group comparison.

women $(89.3 \%, \mathrm{n}=243)$ worked in institutions with a school of medicine affiliation, significantly more female CEs held no academic appointment (men 27.3\%, $\mathrm{n}=56$, women 50.8\%, $\mathrm{n}=34, \mathrm{p}<0.001)($ table 3$)$.

\section{Perceptions of work distribution}

There were no significant gender differences in how CEs spend their time. CEs reported spending approximately half their time (women $54.5 \%$, men $56.4 \%, \mathrm{p}=0.53$ ) providing direct patient care, about a fifth of their time teaching, $6 \%$ in research, with the remainder devoted to administration and other educational work.

Both genders wanted to spend less time in direct patient care, administration and more time in teaching and research (comparison of mean differences, data not shown). However, female CEs ideally wanted to spend a lower percentage of time in patient care (women 46.9\%, men 52.3\%, p=0.03) and a higher percentage of time doing other educational work (women 9\%, men 5.25\%, $\mathrm{p}=0.03$ ), compared with their male counterparts.

Overall, there were no differences in work distribution between genders. However, female CEs in the surgical specialties reported dedicating more time to patient care, both in actual (66.5\% vs $50.9 \%, \mathrm{p}=0.01$, ) and ideal $(57 \%$ vs $42.9 \%, \mathrm{p}=0.018)$ practice compared with female colleagues in medical and hospital-based specialties; these differences also held true for male surgeons (data not shown). All surgeons also reported spending less time teaching, compared with CEs in medical and hospital-based specialties (women $15.3 \%$ vs $21.5 \%, \mathrm{p}=0.05$, men $16.9 \%$ vs $20.6 \%, \mathrm{p}=0.02)$.

\section{Perception of rewards}

There were no gender differences in CEs' perceptions regarding actual or ideal rewards in the areas of administrative promotion, administrative support, financial rewards and protected time. However, as shown in figure 1 , a greater proportion of female CEs reported that they were 'never' rewarded by academic promotion (women $57.6 \%, \mathrm{n}=38$, men $36.7 \%, \mathrm{n}=72, \mathrm{p}=0.02$ ). For both genders, the top ideal rewards were protected time for educational activities and financial incentives.

\section{Satisfaction and job retention}

Comparing males and female CEs, there were no significant differences in overall job satisfaction with the majority of CEs 'satisfied' or 'very satisfied' (women $53.7 \%, \mathrm{n}=36$, men $60.3 \%$, $\mathrm{n}=120, \mathrm{p}=0.34$ ), and a smaller percentage being 'unsatisfied' or 'somewhat unsatisfied' (women 17.9\%, $\mathrm{n}=12$, men $11.1 \%$, $\mathrm{n}=22, \mathrm{p}=0.15$ ). There were no significant differences in the components of satisfaction, which included work-life balance, work duties, work position and opportunities for career advancement. Although results did not reach statistical significance, more female CEs indicated that they are 'likely' or 'very likely' to remain in academic medicine in the next 5 years (women $82.4 \%, \mathrm{n}=56$, men $74.6 \%, \mathrm{n}=148, \mathrm{p}=0.20$ ), while only a small percentage of CEs indicated that they are 'extremely unlikely' or 'unlikely' to stay in academic medicine in the next 5 years (women 5.9\%, $\mathrm{n}=4$, men 5.6\%, $\mathrm{n}=11, \mathrm{p}=0.92$ ).

In multivariable analysis by gender, for both genders, overall job satisfaction was negatively associated with more than $50 \%$ time spent in clinical care (women aOR $=0.16 ; 95 \% \mathrm{CI} 0.04$ to 0.68 , men aOR $=0.32 ; 95 \% \mathrm{CI} 0.17$ to 0.61 ). However, female CEs who spent more than $50 \%$ of their time in direct patient care activities were less likely to be satisfied or very satisfied with work duties and responsibilities $(\mathrm{aOR}=0.18 ; 95 \% \mathrm{CI} 0.05$ to 0.61 ), work-life balance $(\mathrm{aOR}=0.15 ; 95 \% \mathrm{CI} 0.04$ to 0.57 ) and potential for career advancement $(\mathrm{aOR}=0.24, ; 95 \%$ CI 0.07 to 0.08 ) while this did not hold true for men.

There were also differences in perception by gender for those who received fellowship training in the USA, Canada or Europe. Female CEs with such fellowship training were more likely to be unsatisfied with work duties and responsibilities $(\mathrm{aOR}=0.24 ; 95 \%$ CI 0.06 to 0.91$)$ and reported a lower likelihood of staying in their current country of employment for the next 5 years $(\mathrm{aOR}=0.04 ; 95 \% \mathrm{CI} 0.01$ to 0.27$)$. For male CEs, fellowship training in these locations was negatively associated only with their perceptions of potential for career advancement $(\mathrm{aOR}=0.39 ; 95 \% \mathrm{CI} 0.2$ to 0.75$)$.

Within gender analysis showed that female CEs who felt that they made a difference in medical education globally were less likely to be satisfied with work-life balance $(\mathrm{aOR}=0.11 ; 95 \% \mathrm{CI}$ 0.02 to 0.51$)$, potential career advancement $(\mathrm{aOR}=0.23$; $95 \%$ CI 0.06 to 0.88 ) and reported less interest in remaining in their current country of employment for the next 5 years $(\mathrm{aOR}=0.12 ; 95 \% \mathrm{CI} 0.02$ to 0.72$)$. These associations did not hold true for the male CEs. Female CEs were five times as likely to state that they were 'extremely likely' to stay in their current country during the next 5 years $(\mathrm{aOR}=5.02 ; 95 \% \mathrm{CI} 1.20$ to 21.1) if they were single. Having children under 21 was negatively associated with extreme likelihood of staying in academic medicine $(\mathrm{aOR}=0.15 ; 95 \% \mathrm{CI} 0.03$ to 0.79$)$ for women. Marital status and children were not associated with any outcomes for male CEs.

\section{DISCUSSION}

The issue of gender inequity in academic medicine has been described in the medical literature for decades, though most of this data hails from Western countries. There may be some 

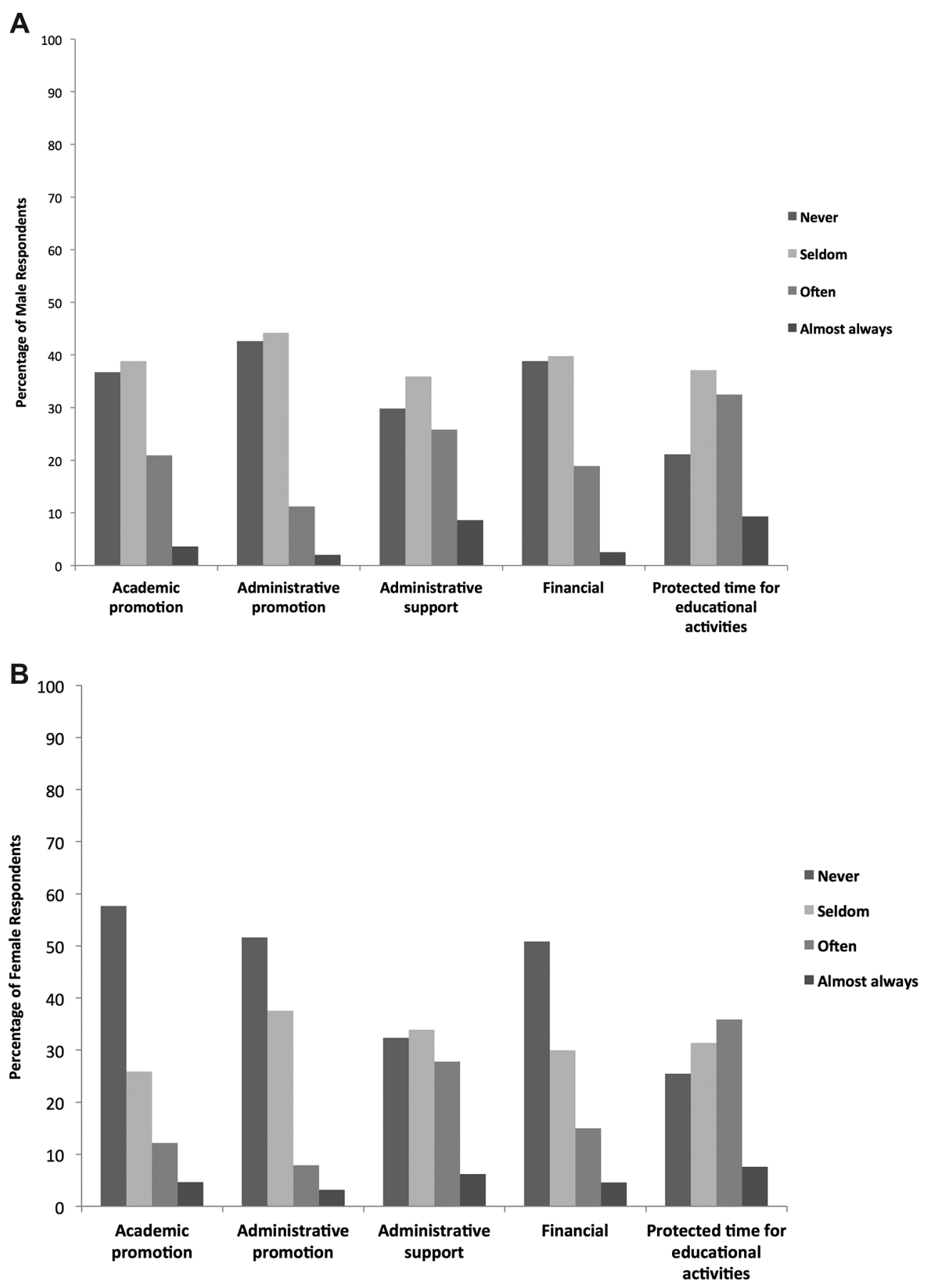

Figure 1 Clinician educators' perception of actual rewards by gender (A) men (B) women.

progress. In the USA, the pipeline has reached gender parity with women comprising almost half of graduating medical student classes. ${ }^{1}$ However, women account for only $38 \%$ of full-time academic faculty and only $21 \%$ of full professors. ${ }^{1}$ Similarly in the UK, while over half of medical school students are women, ${ }^{29}$ women comprise only $29 \%$ of the clinical academic team and only account for $18 \%$ of full professors. ${ }^{30}$ Women also remain significantly under-represented in hospital leadership positions, ${ }^{31}$ comprising only $18 \%$ of hospital CEOs. ${ }^{32}$ Although the percentage of of CEOs was similar to the USA in our study, women comprised only a quarter of the CE workforce and there was only one female CMO. This is concerning as a smaller pipeline of female faculty may have important implications on the gender composition of senior and leadership positions where women are already less represented. Stakeholders in emerging programmes need to develop contextualised strategies to expand the entry of women into academic medicine and decrease potential attrition to meet the challenge of gender equity. Early success has been seen with interventions that educate faculty on hiring and promotion committees about implicit biases and strategies to reduce them. ${ }^{33}$ Mentorship programmes are also essential for career advancement. Yet, women face greater barriers in accessing mentors, with one recent study citing that $34 \%$ of female faculty lack a current mentor and over $10 \%$ have never had a mentor. ${ }^{34}$ Institutions can improve gender equity by developing mentorship programmes for women, with a focus on scholarship and leadership. ${ }^{35}$ Participating in cross-institutional mentorship programmes can also provide junior female 
faculty the opportunity for a better mentor-mentee fit. Additionally, providing opportunities for both formal and informal networking opportunities can create a culture of support for female faculty. One institution, for example, introduced an annual 'Research and Family Life' panel discussion as a formal networking and support initiative. ${ }^{22}$

Similar to other studies, ${ }^{2036}$ we did not find a gender difference in intention to leave academic medicine. Our finding that female faculty had high satisfaction with work-life balance and work duties is promising, as studies have attributed differential career outcomes between men and women, in part, to women faculty facing greater challenges in integrating career and personal responsibilities. ${ }^{18} 2037$ Implementing flexible work-family policies can help academic health centres to better support their female faculty.

It is notable that female CE respondents in our survey were younger, more likely to be single and less likely to have children than their male colleagues and therefore may not have yet faced difficulties balancing work and family life. This is supported by our finding that female respondents with children under age 21 were much less likely to state that they are 'extremely' likely to stay in academic medicine in the next 5 years, implying that other, non-academic factors such as marital status, spouse's career and issues surrounding children may also play a role in women's career and relocation choices. In addition, female CEs who trained abroad were less satisfied and more likely to consider career prospects outside of their current country. With increasing globalisation of medicine and mobility of faculty, programmes will need to offer appealing positions to retain more experienced CE faculty, both male and female.

Notwithstanding the high satisfaction rates, our findings that female CEs are less likely to hold academic appointments as compared with their male colleagues, despite similar time spent in their current position, is concerning. With over half of women in our study reporting no academic appointment and none at the full professor rank, mentorship and role modelling opportunities for female trainees and junior staff may suffer. The culture of academic medicine has been identified as a significant barrier to female faculty advancement, with many studies reporting organisational structure and institutional hierarchy that do not support work-life balance and have a disproportionately negative effect on women faculty satisfaction and retention. ${ }^{4}{ }^{18}{ }^{19}$ Identifying and removing barriers to female CEs' recruitment, initial academic appointment and consequent promotion will be important for programmes to successfully develop and retain their female faculty. This may have already begun; in our study, there were no gender differences in residency-related leadership positions, suggesting a new career path for female CEs. Whether or not this will translate into higher level of leadership remains to be seen.

Our study sites warrant a brief discussion. Although the countries included in this study are in the Middle East and Asia, regions that may traditionally have more conservative views of women in professional life, Singapore, Qatar and the UAE rank highly on the United Nation's Human Development Index, with comparable life expectancy to Western countries and high expected years of education both for men and women. ${ }^{38}$ All these countries have also made significant recent investment in the development of their healthcare and educational infrastructure, in part evidenced by their eagerness to be international pioneers in the transition to $\mathrm{CB}$ education. Though comparisons between countries are not shown to preserve institutional confidentiality, in multivariate analyses, no significant gender differences were seen between study sites. All countries, of course, must take into account their unique sociocultural contexts when planning successful adoption and implementation of gender equity initiatives. Nonetheless, we believe that our findings in these emerging medical education systems, which are consistent with experiences in Western nations, are important and relevant for other international academic institutions evaluating their approach to gender equity, and that our recommendations can be easily adapted to multiple settings.

The strengths of our study include the high response rate and the participation of CEs in emerging GME programmes. CEs represented multiple specialties across a wide array of departments. We attempted to reduce social desirability response bias by assuring respondent, institution and country confidentiality. Our results should be viewed in light of some limitations. First, only programmes of a certain size and that achieved ACGME-I accreditation were included; non-core faculty and those from smaller programmes may have different perceptions. However, we believe that the perceptions of CEs in accredited programmes are a good first impression of the current gender status in these emerging educational systems. Second, bias could have been introduced due to missing respondents. The relatively high response rate is reassuring that our findings accurately represent the demographics and perceptions. Comparison of participants with non-responders did not reveal significant difference in gender, role, department or country. Third, we did not collect salary or productivity dataother factors that impact satisfaction and retention as well as reflect areas of possible gender inequity.

\section{CONCLUSION}

This study is the first, to our knowledge, to explore gender issues for CEs in programmes receiving ACGME-I accreditation, and, more broadly, in international GME. These findings add to the literature on gender equity by identifying disparities in the numbers and academic positions held by women in international GME. Additionally, men disproportionately hold the leadership roles in the international academic medical centres studied. Despite the gender gap, both male and female CEs had high levels of satisfaction and the vast majority intended to remain in academic medicine for the next 5 years. Diversifying faculty recruitment, greater access to institutional resources, structured professional development programmes, skills building workshops, professional networks and mentoring have all been shown to be successful interventions to move towards gender equity. We are optimistic that increased awareness of gender issues in academic medicine will promote efforts to foster inclusivity and equality in the international physician workforce. Given the institutional and societal investment in educating clinical faculty and their role in training the future global physician workforce, realising the full potential of both male and female CEs will only improve the quality of international GME, with the ultimate goal of improving patient care and health outcomes globally.

Main messages

- Only one quarter of the clinician educators (CEs) in the countries studied were female.

- Hospital leadership was male predominant with only $2(18 \%)$ of 11 chief executive officers and $1(9 \%)$ of 11 chief medical officers being women.

- Female CEs were less likely to hold an academic appointment.

- There was no significant difference in overall job satisfaction between genders.

- A number of interventions have been demonstrated to improve gender equity. 


\section{Current research questions}

- To what degree are gender differences present in other international academic settings?

- What are the barriers for female clinician educators in each step of the process-recruitment, initial academic appointment and promotion?

- Which type of interventions would be the most effective in various international settings to eliminate the gender gap?

Acknowledgements The authors wish to thank Amanda Bertram and the Johns Hopkins University School of Medicine, GIM CEMSP, for technical assistance, Abdullatif Alkhal at Hamad Medical Corporation in Qatar and Nicholas Chew at National Healthcare Group in Singapore for their assistance in data acquisition. Finally, the authors extend their gratitude to all the international clinician educators who completed the survey and the graduate medical education office staff in all participating sites.

Contributors DJS and JC had full access to all of the data in the study and take responsibility for the integrity of the data and the accuracy of the data analysis. SA and $\mathrm{HI}$ contributed equally to this manuscript. DJS, HI, SA, JC: study concept and design. HI, SA, DJS, AAAM: acquisition of data. DJS, SA, HI, NGS, JC: analysis and interpretation of data. DJS, SA, HI, NGS, JC: drafting of the manuscript. DJS, SA, HI, NGS, AAAM, JC: critical revision of the manuscript for important intellectual content. DJS, NGS, JC: statistical analysis. DJS, SA, HI, AAAM, JC: Study supervision. All authors, external and internal, had full access to all of the data (including statistical reports and tables) in the study and can take responsibility for the integrity of the data and the accuracy of the data analysis.

Disclaimer DJS affirms that this manuscript is an honest, accurate and transparent account of the study being reported; that no important aspects of the study have been omitted; and that any discrepancies from the study as planned (and, if relevant, registered) have been explained.

Competing interests All authors have completed the ICMJE uniform disclosure form at www.icmje.org/coi_disclosure.pdf and declare no support from any organisation for the submitted work; no financial relationships with any organisations that might have an interest in the submitted work in the previous 3 years; no other relationships or activities that could appear to have influenced the submitted work.

Ethics approval The study received approval from the institutional review boards of Johns Hopkins University School of Medicine in Baltimore, Hamad Medical Corporation and Weill Cornell Medicine in Qatar, National University of Singapore and the Al Ain Research Ethics Committee of the UAE. We also received approval from the GME office of each participating site.

Provenance and peer review Not commissioned; externally peer reviewed.

(c) Article author(s) (or their employer(s) unless otherwise stated in the text of the article) 2017. All rights reserved. No commercial use is permitted unless otherwise expressly granted.

\section{REFERENCES}

1 Lautenberger DM, Dandar VM, Raezet CL, et al. The state of women in academic medicine: the pipeline and pathways to leadership. 2013-2014; www.aamc.org/ publications.

2 McMurray JE, Cohen M, Angus G, et al. Women in medicine: a four-nation comparison. J Am Med Womens Assoc 2002;57:185-90.

3 Carr PL, Gunn CM, Kaplan SA, et al. Inadequate progress for women in academic medicine: findings from the national faculty study. J Womens Health 2015;24:190-9.

4 Abdel-Razig S, Alameri $\mathrm{H}$. Restructuring graduate medical education to meet the health care needs of emirati citizens. J Grad Med Educ 2013;5:195-200.

5 Jagsi R, Guancial EA, Worobey CC, et al. The "gender gap" in authorship of academic medical literature--a 35-year perspective. N Eng/ J Med 2006;355:281-7.

6 Jena $A B$, Khullar D, Ho O, et al. Sex differences in academic rank in US medical schools in 2014. Jama 2015;314:1149-58.

7 Wright AL, Schwindt LA, Bassford TL, et al. Gender differences in academic advancement: patterns, causes, and potential solutions in one US college of medicine. Acad Med 2003;78:500-8.

8 Sandhu B, Margerison C, Holdcroft A. Women in the UK academic medicine workforce. Med Educ 2007;41:909-14.
9 Santamaría A, Merino A, Viñas O, et al. Does medicine still show an unresolved discrimination against women? experience in two european university hospitals. J Med Ethics 2009;35:104-6.

10 Saunders CM, Nichevich A, Ellis C, et al. Frontiers in academic surgery: the five M'S. ANZ J Surg 2008;78:350-5.

11 Sidhu R, Rajashekhar P, Lavin VL, et al. The gender imbalance in academic medicine: a study of female authorship in the united kingdom. J R Soc Med 2009;102:337-42.

12 Larivière $\mathrm{V}, \mathrm{Ni} \mathrm{C}$, Gingras $\mathrm{Y}$, et al. Bibliometrics: global gender disparities in science. Nature 2013;504:211-3.

13 Mayer AP, Blair JE, Ko MG, et al. Gender distribution of U.S. medical school faculty by academic track type. Acad Med 2014;89:312-7.

14 Beasley BW, Wright SM, Cofrancesco J, et al. Promotion criteria for clinician-educators in the united states and Canada. A survey of promotion committee chairpersons. Jama 1997;278:723-8.

15 Fleming VM, Schindler N, Martin GJ, et al. Separate and equitable promotion tracks for clinician-educators. Jama 2005;294:1101-4.

16 Borges NJ, Grover AC, Navarro AM, et al. International women physicians' perspectives on choosing an academic medicine career. Perspect Med Educ 2013;2:156-61.

17 Borges NJ, Navarro AM, Grover AC. Women physicians: choosing a career in academic medicine. Acad Med 2012;87:105-14.

18 Cropsey KL, Masho SW, Shiang R, et al. Why do faculty leave? reasons for attrition of women and minority faculty from a medical school: four-year results. J Womens Health 2008:17:1111-8.

19 Pololi L, Kern DE, Carr P, et al. The culture of academic medicine: faculty perceptions of the lack of alignment between individual and institutional values. J Gen Intern Med 2009;24:1289-95.

20 Pololi LH, Krupat E, Civian JT, et al. Why are a quarter of faculty considering leaving academic medicine? A study of their perceptions of institutional culture and intentions to leave at 26 representative U.S. medical schools. Acad Med 2012;87:859-69.

21 Valantine HA, Grewal D, Ku MC, Mc K, et al. The gender gap in academic medicine: comparing results from a multifaceted intervention for stanford faculty to peer and national cohorts. Acad Med 2014;89:904-11.

22 Athanasiou T, Patel V, Garas G, et al. Mentoring perception, scientific collaboration and research performance: is there a 'gender gap' in academic medicine? An Academic Health Science Centre perspective. Postgrad Med J 2016;92:581-6.

23 Alwazzan L, Rees CE. Women in medical education: views and experiences from the kingdom of saudi arabia. Med Educ 2016;50:852-65.

24 Yasukawa K, Nomura K. The perception and experience of gender-based discrimination related to professional advancement among japanese physicians Tohoku J Exp Med 2014;232:35-42.

25 Al-Tamimi DM. Saudi women in academic medicine. are they succeeding? Saudi Med J 2004;25:1564-7.

26 Ibrahim H, Stadler DJ, Archuleta S, et al. Clinician-educators in emerging graduate medical education systems: description, roles and perceptions. Postgrad Med J 2016;92.

27 Norcini JJ, Mazmanian PE. Physician migration, education, and health care. J Contin Educ Health Prof 2005;25:4-7

28 Krupa C. ACGME extends accreditation abroad to improve physician training. 2012 http://www.amednews.com/article/20121001/profession/310019935/6/ (Accessed May 30, 2014)

29 General Medical Council; Chapter 1: the changing shape of the profession and medical education unknown. http://www.gmc-uk.org/20131004_Chapter_1_SoMEP. pdf_53706030.pdf (accessed 24 Aug 2016).

30 Medical Schools Council; Staffing level of medical clinical academies in UK medical schools, 2015 data. 2016 http://www.medschools.ac.uk/Publications/Pages/Staffingsurvey-2015-data.aspx (accessed 24 Aug 2016).

31 Hoss MAK, Bobrowski P, McDonagh KJ, et al. How gender disparities drive imbalances in health care leadership. J Healthc Leadersh 2011;3:59-68.

32 Rock Health; The state of women in healthcare: 2015. 2015 https://rockhealth.com/ reports/the-state-of-women-in-healthcare-2015/ (accessed 31 May 2016).

33 Girod S, Fassiotto M, Grewal D, et al. Reducing implicit gender leadership Bias in academic medicine with an educational intervention. Acad Med 2016;91:1143-50.

34 Carapinha R, Ortiz-Walters R, McCracken CM, et al. Variability in women faculty's Preferences Regarding Mentor Similarity: A Multi-Institution Study in Academic Medicine. Acad Med 2016;91:1108.

35 Bates C, Gordon L, Travis E, et al. Striving for gender equity in academic medicine careers: a call to action. Acad Med 2016;91:1050-2.

36 Lowenstein SR, Fernandez G, Crane LA. Medical school faculty discontent: prevalence and predictors of intent to leave academic careers. BMC Med Educ 2007;7:37.

37 Pololi LH, Civian JT, Brennan RT, et al. Experiencing the culture of academic medicine: gender matters, a national study. J Gen Intern Med 2013;28:201-7.

38 Human Development Report Office; Human development report 2015: work for human development. 2015; http://report.hdr.undp.org/ (accessed 5 Feb 2017). 